\title{
Hereditary diffuse leucoencephalopathy with spheroids
}

\author{
N Hancock, M Poon, B Taylor, C McLean
}

J Neurol Neurosurg Psychiatry 2003;74:1345-1347

Hereditary diffuse leucoencephalopathy with spheroids (HDLS) is a rare inherited progressive leucoencephalopathy characterised by giant neuroaxonal swellings (spheroids) within the CNS white matter. The case is reported of a 45 year old woman with a rapidly progressive fulminant illness course characterised by progressive cognitive decline with depressive features. A presumed dominant inheritance pattern was elicited. This report reviews the literature on HDLS and the relation of this disorder to other conditions with giant neuroaxonal swellings.

A 45 year old white woman presented with a 16 month history of progressive cognitive decline characterised by depressive symptoms: emotional blunting, lack of spontaneity, lack of initiation, and disturbed diurnal rhythm. The patient had developed the problem of wandering through the house at all hours and required assistance from her family for activities of daily living. An initial psychiatric consultation four months before neurological review suggested a depressive illness; however there was little improvement with the use of a serotonin re-uptake inhibitor for four months. The patient was admitted for neurological assessment because of poor memory, declining cognitive function, insomnolence, distractibility, prominent nocturnal wandering, and urinary and faecal incontinence, which was thought unrelated to a depressive illness. She had not undergone any neurosurgical procedures or received any human derived pituitary hormone therapy. She had travelled to the United Kingdom in the preceding five years.

Physical examination revealed a middle aged woman with no dysmorphic features. Affect was bland with a Folstein MiniMental State examination of 24 of 30, with particular areas of deficit in recall and calculation. Pronounced verbal perseveration was noted. There was no excessive startle response. The gait was broad based and the left arm was held in a flexed posture and was not used in normal activities. A pronounced grasp response was noted in the left hand but no other frontal release signs were noted. Cog wheeling rigidity of the left arm was noted and the left arm would drift downwards. Reflexes were increased on the left with an extensor plantar response on the left. Mild incoordination was noted with left greater than right. Power was preserved throughout. Sensory examination to all modalities was normal. The patient had no clinical evidence for a peripheral neuropathy. There were no skin changes, bony deformities, hepatosplenomegaly, or Kaiser Fleischer rings seen. The patient's hair was straight.

Laboratory investigations were normal and included the following: electrolytes, full blood count, liver function tests, ammonia, thyroid function tests, copper, vitamin B12, folate, syphilis and HIV serology, vitamin E, lactate, amino acids, arylsulfatase $\mathrm{A}$ and galactocerebrosidase in leucocytes, sialic acid, very long chain fatty acids, antinuclear antibody, antineuronal nuclear antibodies, angiotensin converting enzyme, antineutrophil cytoplasmic antibodies, erythrocyte

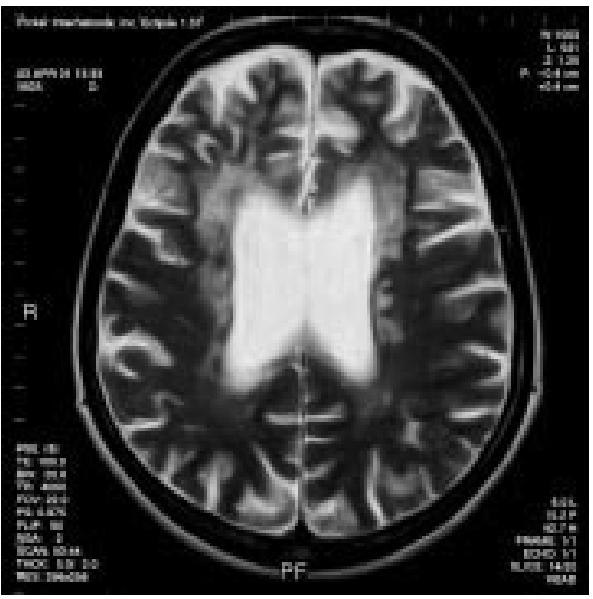

Figure 1 MRI brain T2 weighted image. The image demonstrates cerebral atrophy most marked in the frontal lobes. There is extensive T2 hyperintensity throughout the periventricular white matter, most prominent in the frontal lobes with sparing of the subcortical $U$ fibres.

sedimentation rate. CSF examination was normal for cells, protein, and glucose and negative for brain protein 14-3-3 and oligoclonal bands. Skin and muscle biopsy showed no red ragged fibres, Lafora bodies, or abnormalities of the eccrine sweat glands.

Cranial computed tomography revealed diffuse cerebral atrophy with relative sparing of the cerebellum, confirmed by cranial MRI. The striking feature on MRI was diffuse supratentorial white matter changes on T2 sequences without cortical involvement and with significant cortical atrophy (fig 1). Electroencephalogram was normal. Brain biopsy was considered but declined by the family because of the low chance of detecting a treatable condition.

Over the following months, the patient became increasingly debilitated with worsening cognition, anorexia, spastic dysarthria, markedly dystonic left upper limb and immobility from increasingly painful spasticity in the lower limbs. The family declined invasive feeding methods and death occurred with total illness duration of 21 months from symptoms onset. A postmortem examination of the brain was undertaken.

\section{NEUROPATHOLOGY}

Macroscopical examination of the brain (weight 1370 grams) revealed generalised prominence to sulci. On serial coronal sectioning, cortical sulci appeared prominent with gyri appearing normal. The main changes were that of a patchy irregular pallor to the white matter, sparing the U fibres, associated with prominent dilatation of the lateral and third ventricles. The basal ganglia structure appeared unremarkable. Parasagittal sectioning of the cerebellum and cross sectioning of the brain stem revealed no macroscopic abnormality.

Microscopical examination revealed generalised demyelination of the white matter, with sparing of cortical U fibres. Within the white matter there were frequent axonal balls, 

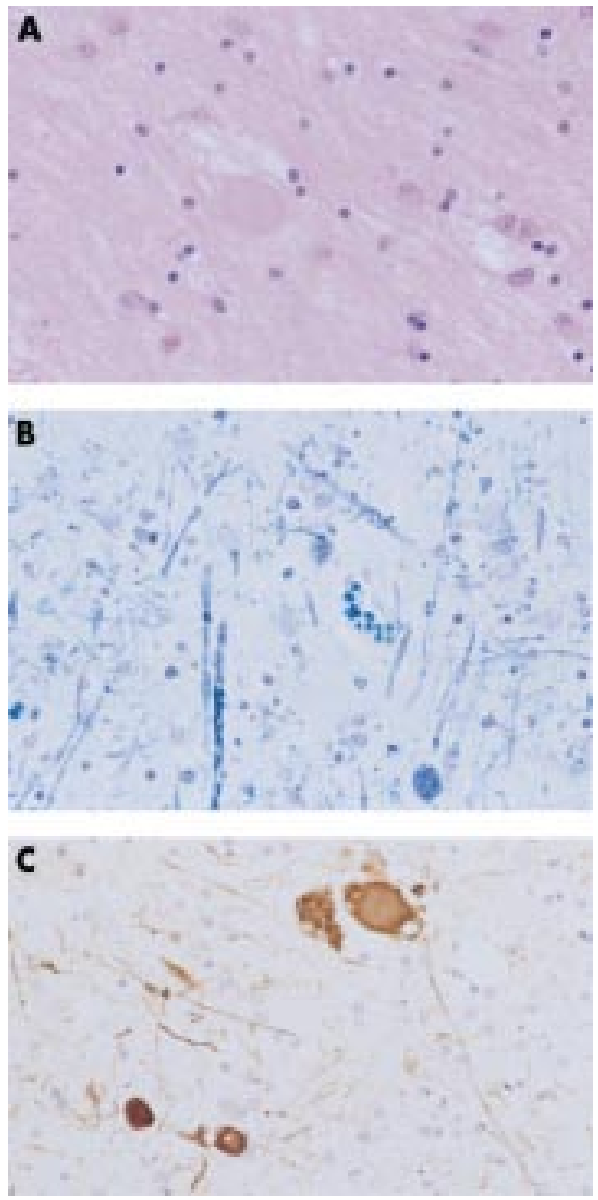

Figure 2 (A) Frontal white matter showing central axonal spheroid with adjacent pallor of myelin and reactive gliosis (haematoxylin and eosin $\times 400$ ). (B) Frontal white matter showing loss of normal myelination with scattered macrophages containing fragmented myelin and residual axons appearing disrupted (luxol fast blue $\times 400$ ). (C) Frontal white matter showing numerous axonal spheroids and disrupted axons (neurofilament (ZYMED) immunoperoxidase $\times 400$ ).

highlighted by ubiquitin and neurofilament immunoperoxidase, associated with axonal loss, and reactive macrophages containing myelin debris (fig $2 \mathrm{~A}, \mathrm{~B}$, and $\mathrm{C}$ ). There was no immunoreactivity with prion protein antibodies (3F4 (Senetek)) or $\alpha$ synuclein (DAKO). No deposition of iron was seen within the sections. There was generalised vacuolation to the white matter. No globoid bodies or an inflammatory cell infiltrate were seen. No inclusions were seen. The overlying cortex appeared comparatively normal. Basal ganglia structures appeared unremarkable although white matter in and around these areas, showed similar changes to that described above, with relative sparing of the mamillothalamic tract. Within the cerebellum minor demyelinating changes were noted in white matter. The cortex was unremarkable. Within the brain stem, descending tracts show demyelination most marked in the corticospinal tract, with relative preservation in the cerebellopontine tracts. The medulla highlighted the loss of myelin in the pyramidal tracts.

The findings were those of a diffuse leucoencephalopathy with neuroaxonal spheroids.

\section{FAMILY HISTORY}

The proband's mother was alive and well, her father had died at age 64 of throat cancer with no history of a dementing illness. However, two paternal siblings, on male and one female, died from a degenerative neurological disorder at ages 66 and
65, respectively. The disorder was clinically diagnosed as Alzheimer's disease. Postmortem material was not available from these family members. No other family members with neurological disease have been reported.

\section{DISCUSSION}

HDLS is rare degenerative neuropsychiatric disorder of presumed dominant inheritance. There is only one recorded patient series in the literature ${ }^{1}$ and a single report of an affected father and daughter. ${ }^{2}$ Isolated cases have been reported and reviewed. ${ }^{2-4}$ Axelsson described a Swedish family with dominant inheritance across four generations with incomplete penetrance and varying neurological presentations and disease course. Age of onset varied between 8 and 60 with psychiatric symptoms and dementia the most prominent neurological features. As occurred in our case, rapid fulminant neurological death was noted; conversely some patients displayed slowly progressive dementia over decades as may have occurred in the probands paternal siblings. In the non-fulminant setting, a diagnosis of Alzheimer's disease would be tenable. In the Swedish cohort, four cases underwent neuropathological examination with all displaying prominent diffuse leucoencephalopathy with myelin loss particularly affecting the frontal, parietal and temporal regions that was thought to correlate well with the clinical manifestations of the disease. The neuropathological findings in the Swedish cases and our case show the same findings of neuroaxonal spheroids associated with lipid laden macrophages and gliosis.

A large proportion of leucoencephalopathies remain unclassified. The term leucoencephalopathy refers to all white matter diseases, both inherited and acquired whereas leucodystrophy refers to progressive, inherited demyelinating disorders. Leucodystrophies presenting in childhood tend to be autosomal recessive or X linked inherited whereas those presenting in adult life are often autosomal dominant. To this regard, HDLS is referred to as a leucoencephalopathy as the genetic and biochemical defects remain to be fully defined.

Spheroids represent swollen, dystrophic axons. On electron microscopy, the axonal swellings have been shown to contain large numbers of neurofilaments, dense bodies, mitochondria, and vesicular profiles. ${ }^{5}$ There have been other reports of the combination of these spheroids and leucoencephalopathy: membranous lipodystrophy or Nasu-Hakola disease, ${ }^{67}$ is a condition characterised by cerebral white matter disease, thalamic degeneration, bone disease, and convulsions. Another disease, dermatoleucodystrophy with neuroaxonal spheroids has combined neurological and dermatological features. ${ }^{8}$ It is of infantile onset and characterised by progressive generalised mental and motor impairment, wrinkly skin and death within two to three years. Features of these disorders are clearly distinct from the clinical, imaging, and pathological findings in our case. Spheroids have also been seen in rats poisoned with parabromophenylacetylurea" and in vitamin E deficiency, usually in association with chronic malabsorption such as seen in cystic fibrosis. ${ }^{10}$

Neuropathological features aside, there is a huge differential for cases of presenile dementia. Examples are X linked adrenoleucodystrophy with frontal predominance, late onset metachromatic leucodystrophy and cerebral autosomal dominant arteriopathy with subcortical infarcts. If clinical and less invasive investigations do not yield a diagnosis, neuropathological features remain the gold standard in undifferentiated cases of presenile dementia.

In the peripheral nervous system, giant axonal neuropathy is a well recognised paediatric condition. ${ }^{11}{ }^{12}$ In a significant proportion of giant axonal neuropathy patients, a degenerative, progressive CNS disorder is superimposed on the peripheral neuropathy. There is diffuse involvement of the CNS white matter with prominent spheroids. ${ }^{13}$ MRI studies have revealed 
features of leucodystrophy. ${ }^{14}$ Most cases of giant axonal neuropathy are thought to be autosomal recessive and present in early childhood with death occurring within the first two decades of life. Recently, the genetic abnormality associated with giant axonal neuropathy has been determined. ${ }^{15}$ Bomont et al found one frame shift and multiple nonsense and missense mutations in the giant axonal neuropathy gene that codes for a ubiquitous protein they termed gigaxonin. This is a member of the cytoskeletal BTB/Kelch repeat family, and is involved in the generalised disorder of cytoskeletal intermediate neurofilaments as seen in giant axonal neuropathy. A novel mutation of this protein may produce the changes seen in our case although this has not been studied.

As previously described, Nasu-Hakola disease has similar neuropathological findings to HDLS. In a recent cohort of Japanese patients with Nasu-Hakola disease mutations of the DAP12 gene were implicated in five of the six cases. ${ }^{16}$ This is in keeping with findings in a group of Finnish patients with Nasu-Hakola disease. ${ }^{17}$ The DAP12 gene encodes for a protein whose function and role in the development of Nasu-Hakola disease has not been established. Characterisation of the pathological pathway in Nasu-Hakola disease and the role of the DAP12 protein may assist us in furthering our knowledge in HDLS.

We report the clinical and pathological findings in a case of HDLS with a presumed dominant inheritance pattern. This adds to the sparse literature available on this disorder. An underlying mutation for this condition is currently unknown. Further families identified and studied through international brain banking and cooperative studies, may help reveal the genetic mutation. We raise the possibility that similarities between the neuropathological changes seen in giant axonal neuropathy and HDLS, may make gigaxonin a potential protein for studies where appropriate material is available.

\section{ACKNOWLEDGEMENTS}

The authors would like to thank the probands family for their consent in writing this report.

\section{Authors' affiliations}

N Hancock, Department of General Internal Medicine, Royal Hobart Hospital, Tasmania, Australia

M Poon, Department of Neurology, Western Hospital, Melbourne, Australia

B Taylor, Department of Neurology, Royal Hobart Hospital

C McLean, The National Health and Medical Research Council
Dementia Brain Bank, The University of Melbourne and The Alfred Hospital, Melbourne, Australia

Correspondence to: Dr N Hancock, Department of General Internal Medicine, Royal Hobart Hospital, GPO Box 1061L, Hobart 7001, Tasmania, Australia; nicole.hancock@dhhs.tas.gov.au

Received 27 October 2002

Accepted in revised form 21 March 2003

\section{REFERENCES}

1 Axelsson R, Roytta $M$, Sourander $P$, et al. Hereditary diffuse leukoencephalopathy with spheroids. Acta Psychiatr Scand 1984;69 (suppl 314):7-65

2 Van der Knapp MD, Naidu S, Kleinschmidt-DeMasters BK, et al. Autosomal dominant diffuse leukoencephalopathy with neuroaxonal spheroids. Neurology 2000:54:463-8.

3 Goodman LA, Dickson DW. Nonhereditary diffuse leukoencephalopathy with spheroids presenting as early-onset, rapidly-progressive dementia. [Abstract]. J Neuropathol Exp Neurol 1995;54:471.

4 Yazawa I, Nakano I, Yamada $\mathrm{H}$, et al. Long tract degeneration in familial leukodystrophy with prominent spheroids. J Neurol Sci 1997:147:185-91.

5 Pentschew A, Schwarz K. Systemic axonal dystrophy in vitamin E deficient adult rats with implications in human neuropathology. Acta Neuropathol (Berl) 1962;1:313-34.

6 Miyazu K, Kobayashi K, Fukutani Y, et al. Membranous lipodystrophy (Nasu-Hakola disease) with thalamic degeneration: report of an autopsied case. Acta Neuropathol 1991;82:414-19.

7 Minagawa M, Maeshiro H, Shioda K, et al. Membranous lipodystrophy (Nasu disease): clinical and neuropathological study of a case. Clin Neuropathol 1985;4:38-45.

8 Matsuyama H, Watanabe I, Mihm MC, et al. Dermatoleukodystrophy with neuroaxonal spheroids. Arch Neurol 1978;35:329-36.

9 Blakemore WF, Cavanagh JB. "Neuroaxonal dystrophy" occurring in an experimental "dying back" process in the rat. Brain 1969;92:789-804.

10 Rosenblum JL, Keating JP, Prensky AL, et al. A progressive neurological syndrome in children with chronic lung disease. N Engl J Med $1981 ; 304: 503-8$

11 Tandan R, Little BW, Emery ES, et al. Childhood giant axonal neuropathy; case report and review of the literature. J Neurol Sci 1987:82:205-28.

12 Donaghy M, Brett EM, Ormerod IE, et al. Giant axonal neuropathy: observations on a further patient. J Neurol Neurosurg Psychiatry 1988;61:991-4

13 Kretzschmar HA, Berg BO, Davis RL. Giant axonal neuropathy: a neuropathological study. Acta Neuropathol 1987;73:138-44.

14 Richen P, Tandan R. Giant axonal neuropathy: progressive clinical and radiological central nervous system involvement. Neurology 1992;42:2220-2.

15 Bomont P, Cavalier L, Blondeau F, et al. The gene encoding gigaxonin a new member of the cytoskeletal BTB/Kelch repeat family, is mutated in giant axonal neuropathy. Nat Genet 2000;26:370-4.

16 Kondo T, Takahashi K, Kohara N, et al. Heterogeneity of presenile dementia with bone cysts (Nasu-Hakola disease). Three genetic forms. Neurology 2002;59: $1105-7$.

17 Paloneva J, Kestila M, Wu J, et al. Loss of function mutations in TYROBP (DAP12) result in a presenile dementia with bone cysts. Nat Genet 2000;25:357-61. 